\title{
Understanding guided light modes in oxide nanoparticles with monochromated EELS
}

\author{
Qianlang Liu ${ }^{1}$, Steven C. Quillin ${ }^{2}$, David J. Masiello² and Peter A. Crozier ${ }^{1}$ \\ ${ }^{1}$ School for the Engineering of Matter, Transport and Energy, Arizona State University, 501 E. \\ Tyler Mall, Tempe, AZ 85287-6106, USA. \\ ${ }^{2}$ Department of Chemistry, University of Washington, Seattle, Washington 98195-1700, USA
}

The ultra-high energy resolution and significantly suppressed zero loss peak tail from monochromated electron energy-loss spectroscopy (EELS) offer opportunities to extract information on semiconductors and insulators from features in the bandgap regions of the spectrum. Intensity variations observed in the bandgap region can be caused by: (1) bandgap states induced by defects or surface adsorbates, and/or (2) phenomena such as Cherenkov radiation and guided light or cavity modes [1]. While bandgap states are of great interest in the field of catalysis and solid state physics, it is also important to understand the origin and behavior of cavity modes in oxide nanoparticles, to differentiate the two thus better interpret the spectra. $\mathrm{TiO}_{2}$ nanoparticles act as an ultraviolet (UV) light photocatalyst for water remediation or solar water splitting, and the surface electronic structure controls the thermodynamics and kinetics of the catalytic reactions. By positioning the focused electron beam in a scanning transmission electron microscope (STEM) a few nanometers outside the particle surface (aloof beam geometry), the electron beam damage can be reduced while obtaining an EELS signal from the surfaces. Interestingly, cavity modes show up in these aloof spectra as intensity maxima in the bandgap region of $\mathrm{TiO}_{2}$ anatase. To further investigate the size and refractive index dependence of the cavity modes in oxide nanoparticles, spectra were also recorded from $\mathrm{CeO}_{2}$ and $\mathrm{MgO}$ nanocubes with simpler more well-defined morphologies. Classical electrodynamic modeling was applied to interpret the bandgap features.

Commercial $\mathrm{TiO}_{2}$ anatase particles (Sigma-Aldrich), hydrothermally synthesized $\mathrm{CeO}_{2}$ cubes and $\mathrm{MgO}$ smoke particles were used for the experiments with refractive indices at $2.5 \mathrm{eV}$ of $2.7,2.5$ and 1.7. An aberration-corrected NION UltraSTEM100 microscope coupled with a monochromator and a Gatan Enfinium spectrometer was employed to acquire all EEL spectra. The microscope was operated at $60 \mathrm{kV}$ and $40 \mathrm{kV}$ with an energy dispersion of $5 \mathrm{meV}$ per channel. The FWHM of the zero-loss peak was less than $25 \mathrm{meV}$. The convergence angle $\alpha$ was $30 \mathrm{mrad}$ and the detector collection angle $\beta$ was $15 \mathrm{mrad}$ with $1 \mathrm{~mm}$ EELS entrance aperture.

Fig. 1a demonstrates the cavity modes detected from an aggregate of anatase nanoparticles. A series of complex peaks were present in the bandgap region in an aloof spectrum where the beam was positioned $4 \mathrm{~nm}$ away from a $\{103\}$ surface recorded with $60 \mathrm{kV}$ electrons. Interestingly, decreasing the voltage to $40 \mathrm{kV}$ (corresponding to a $16 \%$ of beam velocity drop) significantly reduced the overall intensities of these cavity modes. In addition, particle size also plays a role in supporting the cavity modes. As seen in Fig. 1b, the aloof spectrum from an $80 \mathrm{~nm} \mathrm{CeO}$ cube showed a featureless bandgap before the bandgap onset at about $3.2 \mathrm{eV}$. However, a series of bandgap peaks appeared in the aloof spectrum of a cube roughly $250 \mathrm{~nm}$ in size when the electron beam was positioned $4 \mathrm{~nm}$ away from one corner of the cube. In general, the bandgap peaks become sharper and narrower as the energy losses increase. To interpret these spectra, classical electrodynamic simulations were performed on a $250 \mathrm{~nm}$ cube tilted 10 degrees off the (100) zone 
axis. The small discrepancies in the peak energies and strengths between simulation and experiment may be due to imperfect $\mathrm{CeO}_{2}$ dielectric data from literature and an incomplete understanding of the $\mathrm{CeO}_{2}$ nanoparticle's morphology. The spectra from $\mathrm{MgO}$ showed little evidence of cavity modes because of its much lower refractive index. In conclusion, both experiment and simulation show that the energy and strength of the cavity modes depend upon the material's refractive index, particle size and shape, and speed of the fast electron, as well as the beam position relative to the particle. In practice, the cavity modes can be suppressed or eliminated in smaller oxide nanoparticles, enabling the detection of the bandgap states in the materials.

\section{References:}

[1] Q. Liu et al., Ultramicroscopy (in press).

[2] Funding from DOE (DE-SC0004954), NSF CHE-1253775 and the use of NION microscope at John M. Cowley Center for Microscopy at Arizona State University are greatly acknowledged.
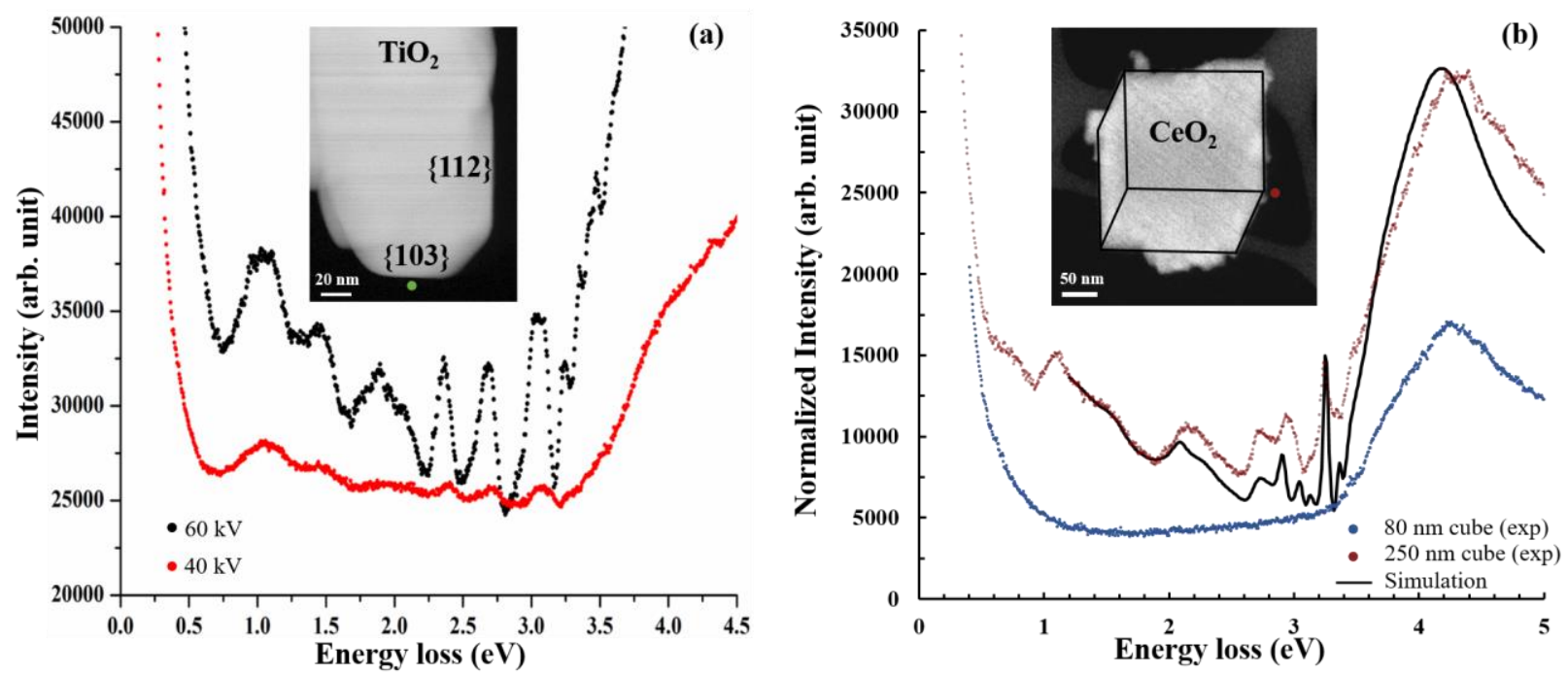

Figure 1. (a) Aloof EELS spectra acquired at 60 and $40 \mathrm{kV}$ from the same facet of a $\mathrm{TiO}_{2}$ particle showing the change in the strength of the bandgap peaks. Inset is an $\mathrm{ADF}$ image of the $\mathrm{TiO}_{2}$ particle with beam position. (b) Aloof EEL spectrum from a small $\mathrm{CeO}_{2}$ cube $(80 \mathrm{~nm})$ showing a featureless bandgap and an aloof EEL spectrum from a large $\mathrm{CeO}_{2}$ cube $(\sim 250 \mathrm{~nm})$ showing intensity variations in the bandgap. Inset shows the ADF image of the large cube with corresponding EELS beam position. The simulated spectrum for the large cube is overlaid on the experimental spectrum. 